\title{
A Survey of the Awareness and Educational Needs of Nurses in Nagasaki Prefecture Regarding Hereditary Breast and Ovarian Cancer
}

\author{
Megumi Matsumoto $^{1}$ (1) $\cdot$ Noriko Sasaki $^{2} \cdot$ Yayoi Tsukigawa $^{3} \cdot$ Ryota Otsubo $^{1} \cdot$ Hiroshi Yano $^{1} \cdot$ Takeshi Nagayasu $^{1}$
}

Accepted: 2 January 2022 / Published online: 6 January 2022

(c) The Author(s) 2022

\begin{abstract}
The aim of this study was to evaluate the knowledge and educational needs with regard to hereditary breast and ovarian cancer among nurses working in breast cancer care in the Nagasaki Prefecture. In breast cancer care, the identification of patients at risk for hereditary breast and ovarian cancer is necessary for the implementation of genetic testing and counseling. Nurses should be involved in this process, since they play a crucial role in the care of patients with breast cancer. However, the knowledge regarding hereditary breast and ovarian cancer among nurses working in oncology care in Japan has not been assessed. The design of this study is cross-sectional design. We distributed 597 surveys to nurses working in breast cancer care. The surveys assessed the nurses' demographic data, their current knowledge and practices regarding cancer genetics and hereditary breast and ovarian cancer, and their attitude and preferences regarding learning about the condition. We received 317 valid replies. Nurses had limited knowledge about hereditary breast and ovarian cancer characteristics: $41.6 \%$ reported that they do not know about the condition, whereas less than $10 \%$ knew its characteristics. However, nurses were aware of hereditary breast and ovarian cancer significance and were willing to learn about it: $91 \%$ wished to learn about the condition, and $88.6 \%$ wanted to participate in study group meetings. Further, nurses' preferences regarding educational programs were clarified. Overall, our results show that educational programs should be implemented to advance nurses' knowledge of hereditary breast and ovarian cancer characteristics.
\end{abstract}

Keywords Educational program $\cdot$ Genetic screening $\cdot$ Hereditary breast and ovarian cancer $\cdot$ Oncology nurses

\section{Introduction}

Breast cancer is one of the most prevalent cancer types worldwide [1]. Hereditary breast and ovarian cancer (HBOC) syndrome is an inherited genetic condition associated with an increased risk of breast and ovarian cancer. Most cases of HBOC are caused by mutations in the breast cancer 1 (BRCA1) or breast cancer 2 (BRCA2) genes [2,

Megumi Matsumoto

mmatsumoto@nagasaki-u.ac.jp

1 Department of Surgical Oncology, Nagasaki University Graduate School of Biomedical Sciences, 1-7-1 Sakamoto, Nagasaki City, Nagasaki 852-8501, Japan

2 Department of Reproductive Health, Institute of Biomedical Science, Nagasaki University, Nagasaki City, Nagasaki 852-8501, Japan

3 Department of Nursing, Nagasaki University Hospital, Nagasaki City, Nagasaki 852-8501, Japan
3]. The identification of patients with breast and/or ovarian cancer at high risk for HBOC is important to ensure appropriate genetic testing and counseling. Moreover, there is a high burden of need for genetic testing in patients at risk for HBOC [4]. To ensure adequate screening, genetic testing, and counseling for HBOC, healthcare providers should have sufficient knowledge in the field of cancer genetics and genomics [5]. However, many healthcare professionals do not receive sufficient training in genetics and genetic testing, which increases the risk of errors $[6,7]$.

Nurses play a critical role in the care of patients with breast cancer; therefore, they should be closely involved in screening for patients at high risk for HBOC. However, a survey conducted with Turkish oncology nurses showed that their knowledge of cancer genetics was only moderate $[8,9]$. Similar findings were obtained in a Brazilian study, which found that the majority of nurses had not identified or referred a patient for specialized risk assessment due to a genetic risk [10]. Only a few studies have 
assessed the knowledge of healthcare providers in Japan regarding HBOC screening, testing, and counseling, and none focused on nurses. A previous investigation found that although there was an awareness of the significance of HBOC testing among Japanese healthcare providers, it was generally not sufficiently implemented in practice, and there was a need to further educate nurses. However, that study primarily assessed the knowledge and educational needs of clinical geneticists, other physicians, and genetic counselors, whereas only $2.4 \%$ of the respondents were nurses [11]. Moreover, in a study limited to physicians, genetics training and continuing medical education were associated with better knowledge of HBOC risk assessment [12].

The authors' institution has the capacity to construct a medical care system for HBOC primary screening and identification, risk assessment, genetic counseling, genetic testing, surveillance, and management of risk-reducing surgery. However, even though it is the only institution capable of providing $\mathrm{HBOC}$ care in the prefecture, there have been few cases of genetic counseling (47 cases between 2014 and 2016). Hospitals in the prefecture, including this institution, have not yet succeeded in placing appropriate cases on the care flow as HBOC cases.

In addition to their knowledge of $\mathrm{HBOC}$, the educational needs of nurses working in breast cancer care should also be assessed. The necessity for further genetic education has previously been described among nurses in other settings [13]. However, the knowledge and specific educational needs of nurses working in cancer care in Japan have not been analyzed.

The aim of this study was to gain insight into the level of understanding, interest, and educational needs of nurses regarding HBOC by conducting a survey among nurses working in the field of breast cancer care in Nagasaki Prefecture.

\section{Materials and Methods}

\section{Study Design and Participants}

Nurses working in the field of breast cancer care at 23 institutions in Nagasaki Prefecture were contacted for participation in this descriptive study. The study was approved by the Ethics Committee of the Nagasaki University Hospital. It was conducted between June 1, 2016 and November 30, 2016. A questionnaire was distributed via physicians at each institution, and answers were collected from each respondent by mail. By mailing in their replies, the respondents were deemed to have provided consent. The total number of distributed questionnaires was 597, while the number of valid responses was 317 (53.1\%).

\section{Study Survey}

The survey was developed by the authors of this study and included 14 questions. The first part of the survey (questions 1-4) gathered information about the participants' age, sex, professional qualifications, and current work situation. The second part (questions 5-10) inquired about nurses' current knowledge and practices regarding cancer genetics and HBOC. These questions clarified whether the nurses had been involved in breast cancer care and for how long, whether and to what extent they had collected the family history of patients with breast cancer, whether and to what extent they were aware of HBOC, and whether they had received a question or consultation request regarding genetics by a patient with breast cancer. The third part of the survey (questions 11-14) assessed whether nurses had had the opportunity to learn about HBOC, whether they wanted to learn about it, what their motivation for learning was, and what their preferred learning format was.

\section{Statistical Analysis}

The multiple-choice questions included in the survey were assessed with descriptive statistics, whereas the open-ended questions were evaluated using content analysis.

\section{Results}

\section{Participants' Characteristics}

The majority of the participants were women (97\%). More nurses worked in a ward (70\%) than in an outpatient setting (27\%). The most prevalent duration of experience working with breast care was $1-5$ years ( $47 \%$ of nurses working in wards and $59 \%$ of nurses working in outpatient units) (Supplementary Table S1).

\section{Nurses' Practices with Respect to Family History Assessment}

Slightly more than half of the nurses (52\%) participating in the study reported that they were aware of the family history of patients with cancer. Moreover, $63 \%$ responded that they asked patients with cancer about their family history. However, only $23 \%$ of nurses who recorded a family history also asked about the family history of extended family members up to third-degree relatives (Table 1).

\section{Nurses' Knowledge and Practices with Respect to $\mathrm{HBOC}$ and Its Screening}

When the nurses were asked whether they knew about HBOC, $41.6 \%$ reported that they did not know about it, 
Table 1 Current practices of the nurses with respect to family history

\begin{tabular}{lll}
\hline Questions & Possible answers & Rates \\
\hline Do you ask about the family history of patients? $(n=317)$ & Yes & $63 \%$ \\
& No & $36 \%$ \\
& Unknown & $1 \%$ \\
Do you ask about the family history of the extended family members up to the & Yes & $23 \%$ \\
third-degree relatives? (If the answer to the previous question is yes, $n=199)$ & No & $75 \%$ \\
& Unknown & $2 \%$ \\
When you see a cancer patient, are you aware of their family history? $(n=317)$ & I am aware & $52 \%$ \\
& I am not aware & $48 \%$ \\
\hline
\end{tabular}

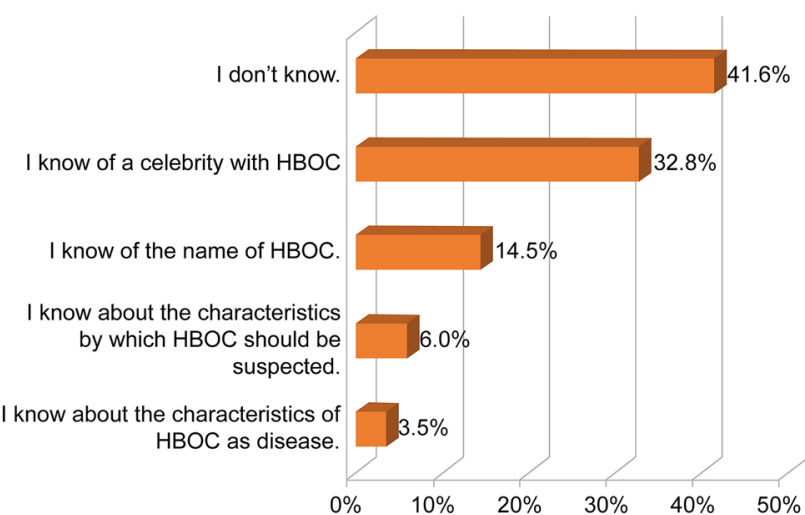

Fig. 1 The degree of nurses' knowledge regarding hereditary breast and ovarian cancer

whereas $58.4 \%$ reported that they did. However, fewer than $10 \%$ of the respondents knew about the characteristics of $\mathrm{HBOC}$ or the characteristics leading to suspicion of HBOC (Fig. 1).

Open-ended questions were also asked about the knowledge and practices of nurses with respect to questions or consultation requests concerning genetics by breast cancer patients (Supplementary Table S2). The most frequently asked question was "Can my close relatives (especially my daughter) inherit the cancer from me?" The most common answer to this question was "I recommended a self-examination and a breast cancer screening test."

\section{Attitudes of Nurses Regarding Learning About HBOC}

Ninety-one percent of the participants in the survey were willing to learn about HBOC; furthermore, $88.6 \%$ of the respondents wished to participate in $\mathrm{HBOC}$ study group meetings. However, before the survey, the majority of respondents had had the opportunity to learn only about breast cancer (62\%) but not about heritability (3\%). The most important reasons for wishing to learn were wanting to utilize this knowledge to provide information when being questioned $(86.6 \%)$ or to implement it in nursing $(53.4 \%)$ (Table 2).

The topics nurses wanted to learn about included the pathology of HBOC (76\%), basic information about inheritance $(68.5 \%)$, the reality of HBOC care $(61.5 \%)$, genetic testing (47.6\%), and genetic counseling (33.1\%). The majority of nurses were interested in hour-long study sessions. The internet $(85.5 \%)$ and journals $(70.3 \%)$ were the most frequently used sources for learning (Table 2).

\section{Discussion}

The current study found that knowledge regarding HBOC among Japanese nurses working in the field of breast cancer care is limited, whereas their interest in learning more about it is pronounced.

In this study, the nurses had limited knowledge of HBOC inheritance. These findings agree with previous publications, which showed limited knowledge of hereditary breast cancer/HBOC among nurses in Brazil and Turkey, especially with respect to genetic counseling $[9,10]$. Our study was based on self-assessment of HBOC knowledge among nurses. However, the utilization of objective assessment tools may provide more reliable information. Such tools should focus on examining nurses' knowledge of cancer genetics and HBOC. A range of factors should be assessed, including knowledge of the risk factors, epidemiology, inheritance patterns, screening, diagnosis, and treatment for HBOC. Similar questionnaires have been developed and have been successfully implemented to assess the knowledge of nurses regarding hereditary breast cancer $[9,10]$. Moreover, future studies should compare HBOC awareness between nurses from cancer centers that have and have not implemented HBOC educational programs.

The nurses were aware of the significance of HBOC awareness and screening. A previous study showed that involving nurses in screening for HBOC could help identify patients who required further testing [14]. Moreover, nurses' knowledge regarding pharmacogenetics and pharmacogenomics in general may improve patient outcomes 
Table 2 Nurses' attitudes regarding learning about inheritance and hereditary breast and ovarian cancer (HBOC)

Questions
Have you had an opportunity to learn about cancer and inherit-
ance? $(n=317)$

Do you wish to learn about HBOC? $(n=317)$

\begin{tabular}{ll} 
Answers & Rates \\
\hline I only had an opportunity to learn about breast cancer & $62 \%$ \\
I only had an opportunity to learn about inheritance & $3 \%$ \\
I had an opportunity to learn about both & $9 \%$ \\
I have had neither learning opportunity & $26 \%$ \\
Yes & $91 \%$ \\
No & $8 \%$ \\
Unknown & $1 \%$ \\
I would like to utilize what I learn to provide information when & $86.6 \%$ \\
being questioned & \\
I would like to implement what I learn in nursing & $53.4 \%$ \\
It is useful for me and people close to me, such as relatives & $31.4 \%$ \\
It is a hot topic these days & $15.2 \%$ \\
I do not feel a particular need to learn & 11 \\
I am not interested & 9 \\
There is no established system yet, etc & 6 \\
HBOC pathology (incidence rates of cancer, inheritance, etc.) & $76.0 \%$ \\
Basic information about inheritance & $68.5 \%$ \\
The reality of HBOC care (identifying patients, asking about the & $61.5 \%$ \\
family history, etc.) & \\
Genetic testing & $47.6 \%$ \\
Genetic counseling & $33.1 \%$ \\
I wish to participate & $88.6 \%$ \\
I do not wish to participate & $7.9 \%$ \\
Unknown & $3.5 \%$ \\
Less than 30 min & $12.5 \%$ \\
1 h & $62.1 \%$ \\
2 h & $12.8 \%$ \\
Over 2 h & $1 \%$ \\
Unknown & $11.6 \%$ \\
Sht & \\
\hline
\end{tabular}

Wishes for the study group meetings (open-ended)

Short duration/multiple times/serialization

e-Learning/on-demand/live

Inclusion of physicians

Provision of documents

Q\&A of frequently asked questions

Held on weekends/in the evenings after work

How do you check on what you do not know during nursing work?

[15]. Therefore, the results of the present and previous studies indicate that genetics and genomics knowledge is critical for oncology nurses [16].

The current study found that nurses are motivated to learn about HBOC characteristics, mainly to answer patients' questions and to implement the newly acquired knowledge in nursing. Previous studies also found that the majority of nurses are motivated to continue their education in the field of cancer genetics $[9,10]$.
The development of web-based educational interventions in the fields of genetics and genomics is an area of active research [17]. Standards for the core competencies of healthcare professionals in the field of genomic medicine have been established to serve as a basis for the development of educational programs [18]. These standards relate to the domains of knowledge, attitudes, and abilities [5]. Educational genetics interventions, including interactive workshops and PowerPoint modules, have also been developed 
and have shown to be effective in educating primary healthcare providers [19]. In this survey, the nurses reported that they used the internet as a primary source of learning. In Nagasaki Prefecture, there are many minor islands, and interactive study group meetings via e-learning and internet are being considered by our team and institution, including in remote areas.

Promoting nurses' knowledge of cancer genetics will also have beneficial effects on patient education. For example, education for breast self-examination of women and $B R C A 1$ or BRCA2 mutations has been proven to be feasible [20].

This study has several limitations. First, it used self-report measures to assess nurses' awareness of HBOC. These findings should be confirmed through more objective assessments. Second, it did not directly evaluate the effect of nurses' knowledge regarding HBOC on the effectiveness of patient screening and the identification of patients at high risk for HBOC. Third, even though it assessed nurses' preferences regarding the development of an educational program, further studies should determine the optimal design of the program.

\section{Conclusion}

The results of this awareness survey among nurses working in breast cancer care institutions in Nagasaki Prefecture indicate that most nurses do not know about the disease characteristics and inheritance patterns of HBOC but are eager to learn about them. Therefore, further focused learning opportunities should be provided to nurses working in breast cancer care.

Supplementary Information The online version contains supplementary material available at https://doi.org/10.1007/s13187-022-02132-4.

Acknowledgements We thank the institutions that participated in this study: Imamura Women's Clinic, Inoue Hospital, Isahaya General Hospital, Itoyanagi Breast Clinic, Izumikawa Hospital, Kobayashi Hospital, Kouseikai Hospital, Menoto Clinics, Nagasaki Ekisaikai Hospital, Nagasaki Genbaku Hospital, Nagasaki Harbor Medical Center, Nagasaki Kamigoto Hospital, Nagasaki Kawatana Medical Center, Nagasaki Medical Center, Nagasaki Rosai Hospital, Nagasaki University Hospital, Nijigaoka Hospital, Sasebo City General Hospital, Sasebo Chuo Hospital, Saiseikai Nagasaki Hospital, St. Francis Hospital, Uga Surgical Clinic, and Yutaka Fukuda Clinic. Editorial support, in the form of medical writing, table assembly, and creation of high-resolution images based on the authors' detailed directions, collation of author comments, copyediting, fact checking, and referencing, was provided by Editage, Cactus Communications.

Author Contribution Megumi Matsumoto: Conceptualization and formal analysis

Noriko Sasaki: Methodology and formal analysis

Yayoi Tsukigawa: Investigation and data curation

Hiroshi Yano: Investigation

Ryota Otsubo: Investigation
Takeshi Nagayasu: Supervision

Data Availability All data generated or analyzed during this study are included in this published article (and its supplementary information files).

Code Availability Not applicable.

\section{Declarations}

Ethics Approval Nurses working in the field of breast cancer care at 23 institutions in Nagasaki Prefecture were contacted for participation in this descriptive study. The study was approved by the Ethics Committee of the Nagasaki University Hospital.

Consent to Participate A questionnaire was distributed via physicians at each institution, and answers were collected from each respondent by mail. By mailing in their replies, the respondents were deemed to have provided consent.

Consent for Publication The consent for publication was not required as no intervention occurred.

Conflict of Interest The authors declare no competing interests.

Open Access This article is licensed under a Creative Commons Attribution 4.0 International License, which permits use, sharing, adaptation, distribution and reproduction in any medium or format, as long as you give appropriate credit to the original author(s) and the source, provide a link to the Creative Commons licence, and indicate if changes were made. The images or other third party material in this article are included in the article's Creative Commons licence, unless indicated otherwise in a credit line to the material. If material is not included in the article's Creative Commons licence and your intended use is not permitted by statutory regulation or exceeds the permitted use, you will need to obtain permission directly from the copyright holder. To view a copy of this licence, visit http://creativecommons.org/licenses/by/4.0/.

\section{References}

1. Harbeck N, Penault-Llorca F, Cortes J, Gnant M, Houssami N, Poortmans P, Ruddy K, Tsang J, Cardoso F (2019) Breast cancer. Nat Rev Dis Primers 5(1):66

2. Petrucelli N, Daly MB, Pal T (1998) [updated 2016 Dec 15]. BRCA1- and BRCA2-associated hereditary breast and ovarian cancer. In: Adam MP, Ardinger HH, Pagon RA, Wallace SE, Bean LJH, Mirzaa G, Amemiya A (eds) GeneReviews ${ }^{\circledR}$ [Internet]. University of Washington, Seattle; pp: 1993-2021

3. Yoshida R (2020) Hereditary breast and ovarian cancer (HBOC): review of its molecular characteristics, screening, treatment, and prognosis. Breast Cancer. https://doi.org/10.1007/ s12282-020-01148-2

4. Singh N, Shukla S, Agrawal S, Singh U, Kumar V (2019) Assessment of high risk of hereditary breast and ovarian cancer (HBOC) and acceptance for genetic testing among cases of ovarian and breast cancer in Indian set-up. Indian J Gynecol Oncol 17:35

5. Tognetto A, Michelazzo MB, Ricciardi W, Federici A, Boccia S (2019) Core competencies in genetics for healthcare professionals: results from a literature review and a Delphi method. BMC Med Educ 19(1):19 
6. Brierley KL, Blouch E, Cogswell W, Homer JP, Pencarinha D, Stanislaw CL, Matloff ET (2012) Adverse events in cancer genetic testing: medical, ethical, legal, and financial implications. Cancer J 18(4):303-309

7. Nair N, Bellcross C, Haddad L, Martin M, Matthews R, GabramMendola S, Crane B, Meaney-Delman D (2017) Georgia primary care providers' knowledge of hereditary breast and ovarian cancer syndrome. J Cancer Educ 32(1):119-124

8. Seven M, Akyüz A, Elbüken B, Skirton H, Öztürk H (2015) Nurses' knowledge and educational needs regarding genetics. Nurse Educ Today 35(3):444-449

9. Seven M, Pasalak SI, Guvenc G, Kok G (2017) Knowledge level and educational needs of Turkish oncology nurses regarding the genetics of hereditary breast and ovarian cancer. J Contin Educ Nurs 48(12):570-576

10. Prolla CM, da Silva PS, Netto CB, Goldim JR, Ashton-Prolla P (2015) Knowledge about breast cancer and hereditary breast cancer among nurses in a public hospital. Rev Lat Am Enfermagem 23(1):90-97

11. Terui-Kohbata H, Yoshida M (2017) Current condition of genetic medicine for hereditary breast cancer. Mol Clin Oncol 7(1):98-102

12. Cohn J, Blazey W, Tegay D, Harper B, Koehler S, Laurent B, Chan V, Jung MK, Krishnamachari B (2015) Physician risk assessment knowledge regarding BRCA genetics testing. J Cancer Educ 30(3):573-579

13. Chair SY, Waye MMY, Calzone K, Chan CWH (2019) Genomics education in nursing in Hong Kong, Taiwan and Mainland China. Int Nurs Rev 66(4):459-466

14. Appel SJ, Cleiment RJ (2015) Identifying women at risk for hereditary breast and ovarian cancer syndrome utilizing breast care nurse navigation at mammography and imaging centers. $\mathbf{J}$ Natl Black Nurses Assoc 26(2):17-26

15. Dodson CH (2017) Pharmacogenomics: principles and relevance to oncology nursing. Clin J Oncol Nurs 21(6):739-745

16. Eggert J (2017) Genetics and genomics in oncology nursing: what does every nurse need to know? Nurs Clin N Am 52(1):1-25

17. Dumo AM, Laing B, Lim AG, Palaganas E, Abad PJ, Valdehueza O, Palovaara M, Saunders H, Estola M, Mandysova P, Maguire J, Ward LD, Carlberg C, Vehviläinen-Julkunen K (2020) Randomized controlled trial on the effectiveness of web-based Genomics Nursing Education Intervention for undergraduate nursing students: a study protocol. J Adv Nurs 76(11):3136-3146

18. Skirton H, Lewis C, Kent A, Coviello D (2010) Genetic education and the challenge of genomic medicine: development of core competences to support preparation of health professionals in Europe. Eur J Hum Genet 18(9):972-977

19. Carroll JC, Rideout AL, Wilson BJ, Allanson JM, Blaine SM, Esplen MJ, Farrell SA, Graham GE, MacKenzie J, Meschino W, Miller F, Prakash P, Shuman C, Summers A, Taylor S (2009) Genetics education for primary-care providers: improving attitudes knowledge and confidence. Can Fam Physician 55:e92-99

20. Visser A, Bos WC, Prins JB, Hoogerbrugge N, van Laarhoven HW (2015) Breast self-examination education for BRCA mutation carriers by clinical nurse specialists. Clin Nurse Spec 29(3):E1-7

Publisher's Note Springer Nature remains neutral with regard to jurisdictional claims in published maps and institutional affiliations. 